\title{
Study of Routing Algorithms in DTN Enabled Vehicular Ad-Hoc Network
}

\author{
Rakesh Kumar Agarwal \\ M.Tech. Scholar \\ Department of CSE, Maharishi \\ Arvind College of Engg. \& R.C. \\ aipur, Rajasthan
}

\author{
Manish Mathuria \\ Assistant Professor \\ Department of CSE, \\ M.A.C.E.R.C. \\ Jaipur
}

\author{
Manish Sharma \\ Assistant Professor \\ Department of CSE, \\ M.A.C.E.R.C. \\ Jaipur
}

\begin{abstract}
Vehicular ad hoc networks (VANETs) are the special type of mobile ad hoc networks (MANETs) that are very frequent network in which partitions are caused by node mobility. Delay tolerant network (DTN) follows the approach to store and forward the message. Delay Tolerant Networks (DTN) are the networks which do not require the immediate data delivery and these networks can wait for a specific time period before transferring of data. VANET introduces lot of challenges because of high mobility of the nodes and fast topology changes in the VANET. Various kind of routing protocols have been designed and presented by the researchers after considering the major challenges that are involved in DTN enabled VANETs. This paper provides a survey of the VANET, DTN and routing protocols for DTN enabled VANET
\end{abstract}

\section{Keywords}

VANET, DTN, VDTN, Routing algorithm, FFRDV.

\section{INTRODUCTION}

Wireless networks have enabled number of devices to be connected over the vast distances [1]. But they still cannot reach everywhere, and for some of the applications, their cost is preventing. Ad hoc networks are infrastructure free, wirelessly connected and distributed systems in which no central administration is available for controlling the different operations in network. Each node has capabilities of a router that helps in providing the multi hop communication among the mobile nodes which do not have direct link. There are some constraints in the ad hoc networks i.e. limited battery backup, radio range, and heterogeneity of devices [2].

There is development in the intelligent transportation systems (ITS). It is due to the human desire for change, progress, mobility, entertainment, safety and security So Vehicular Adhoc network is most suitable technology for ITS. The participating mobile nodes in VANET communication networks (vehicles) interact with each other by short-range direct communications, by anticipating messages through vehicles (Vehicle-to-Vehicle) and road side units [3]. Information about traffic on the road is obtained through inductive loops, roadside sensors and studies. VANETs offer locations for collecting the real time information- from onboard sensors placed on vehicles and its quick distribution. The information collected through every individual vehicles participating in the network now integrated together to form a real time picture of the particular road situation. The various ITS stakeholders like different governments, communication companies and vehicle manufacturers are working together to make the "VANET based ITS" a reality [3].
This paper is organized in five sections. In first section, the introduction is given. In second section, the vehicular adhoc network (VANET) is explained which covers architecture, routing protocols and different scenarios. In third section, delay tolerant network (DTN) is discussed. In fourth section, routing algorithm in Vehicular DTN is discussed. The last fifth section gives the conclusion.

\section{VEHICULAR AD-HOC NETWORK (VANET)}

Vehicular ad hoc networks (VANETs) are different from the basic wireless network because of high mobility and the predictable mobility patterns [2]. Vehicular ad hoc network is very popular area of research in which different researchers from all over the world are devoting their time to provide a safe and enjoyable drive on crowded roads. Communication in VANETs is more challenging due to infrastructure free environment and frequently changes in the network. In VANTEs the nature of motion can be predicted by using the roads and digital city maps [2].

\subsection{System Architecture}

According to the IEEE 1471-2000 and ISO/IEC 42010 the VANETs system can be divided into three domains: the mobile domain, the infrastructure domain, and the generic domain [4].

As is shown in figure 1, the mobile domain also consists of two parts: the vehicle domain and the mobile device domain. The vehicle domain contains all kinds of vehicles like cars and buses. The mobile device domain contains all kinds of portable devices like smart phones.

In the infrastructure domain, there are also two domains: the roadside infrastructure domain and the central infrastructure domain. The roadside infrastructure domain contains roadside unit entities like traffic lights. The central infrastructure domain contains infrastructure management centers like traffic management centers (TMCs) and vehicle management centers. The development of the VANETs architecture varies from region to region. CAR-2-CAR communication consortium $(\mathrm{C} 2 \mathrm{C}-\mathrm{CC})$ is the major driving force for vehicular communication in Europe.

The generic domain contains Internet Infrastructure Domain and Private Infrastructure Domain. 


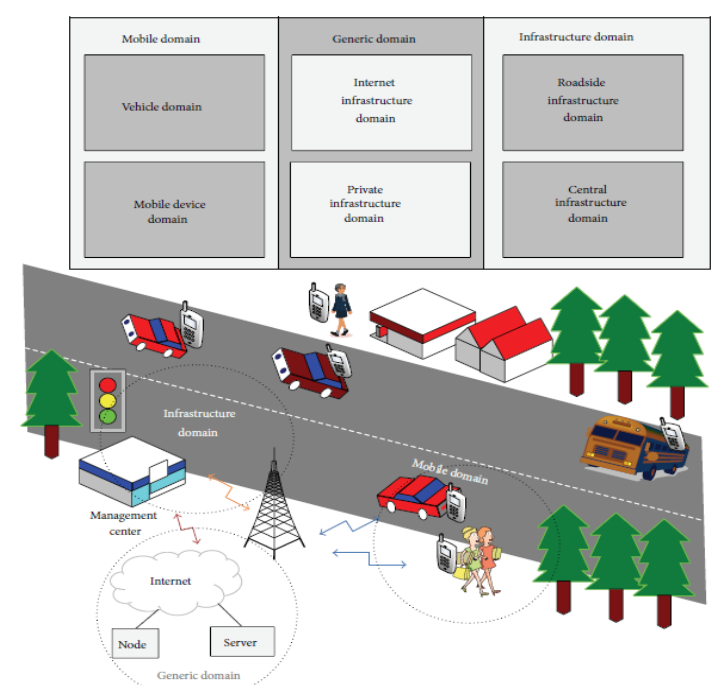

Fig 1: VANETs system domains [4]

This system architecture has three domains: in-vehicle, ad hoc, and infrastructure domain as shown in fig. 2. The invehicle domain is composed of an on-board unit (OBU) and one or multiple application units (AUs).The connections between these are usually wired or wireless. The ad hoc domain is composed of vehicles equipped with OBUs and roadside units (RSUs). An OBU can be considered as a mobile node of an ad hoc network and RSU is a static node. An RSU connected to the Internet via the gateway. RSUs can communicate with each other directly.

There are two types of infrastructure domain access, first is RSUs and second is a hot spot (HS). OBUs may communicate with Internet via RSUs or HSs. In the absence of the RSUs and HSs, the OBUs can communicate with each other by using cellular radio networks.

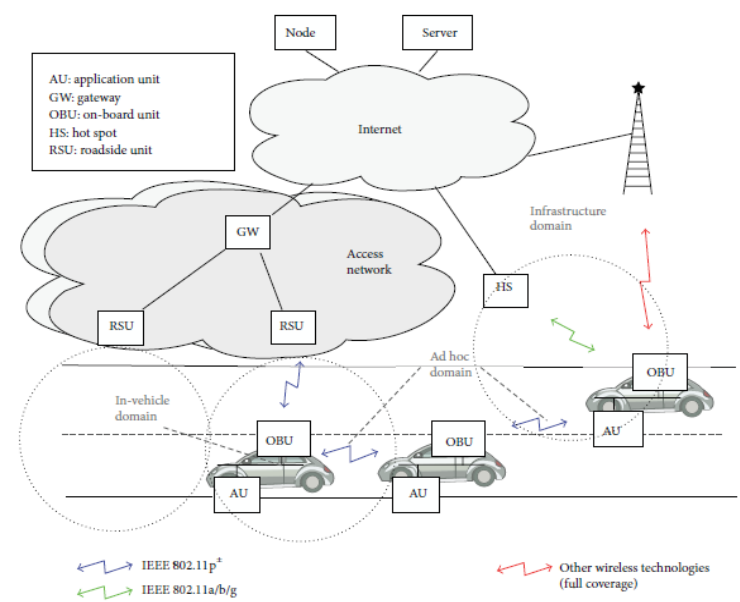

Fig 2: C2C-CC reference architecture [4].

Communication types in VANETs are categorized into four types [4].

In-vehicle communication system can detect the vehicle's performance and specially driver's fatigue and drowsiness, which is the critical factor for driver and public safety.

Vehicle-to-vehicle (V2V) communication provides a data exchange platform for the drivers to share information and warning messages, so that it expands driver's assistance.
Vehicle-to-road infrastructure (V2I) communication enables the real-time traffic/weather updates for drivers of the vehicle and provides environmental sensing and monitoring.

Vehicle-to-broadband cloud (V2B) communication, in which vehicle may communicate each other via wireless broadband mechanisms. This type of communication is useful for active driver assistance and vehicle tracking purpose.

\subsection{Routing}

In VANETs [4], wireless communication is a critical technology to support the achievement of many applications and services. Depending on the number of senders and receivers, routing approaches can be divided into following three types: geocast / broadcast, multicast, and unicast approaches.

2.2.1 Geocast /Broadcast. To distributing the messages to unknown / unspecified destinations, the geocast/broadcast protocols are used in VANETs. In [9], the researchers review the current message broadcast protocols in vehicular ad hoc networks, for example a spatially aware packet routing algorithm, SADV, an interference aware routing scheme, FROV, and a multi hop broadcast protocol. Other researchers also proposed some algorithms such as V-TRADE, UMB, and AMB etc. [4].

2.2.2 Multicast. Multicast is used to communicate among a group of vehicles in some vehicular situations, such as intersections, roadblocks, high traffic density, and dangerous road surface conditions.

In [5], the authors categorize the different multicast protocols into two types. One is topology based approaches, such as ODMRP, MAODV, and GHM. The other one is locationbased approaches, such as PBM, SPBM, LBM, and RBM and IVG.

2.2.3 Unicast. The researchers also investigate the unicast communication protocols for VANETs in following three ways [4]: -

2.2.3.1 Greedy: Nodes forward the packets to their farthest neighbors towards the destination, like improved greedy traffic-aware routing (GyTAR).

2.2.3.2 Opportunistic: Nodes employ the carry-toward technique to opportunistically deliver the data to the destination, like topology-assist geo-opportunistic routing.

2.2.3.3 Trajectory based: Nodes calculate the possible paths to the destination and deliver the data through nodes along one or more of those paths, like trajectory-based data forwarding (TBD)

\subsection{VANET Traffic Scenario}

The different scenario used for the VANET, are as following [7] -

\subsubsection{Highway Scenario}

Highway scenario in the algorithm assumes that number of nodes is less, speed of the nodes is high and vehicles are travelling in the only one direction as shown in fig. 3. Nodes travelling in the opposite direction are not considered for the routing as there is no need for communication because they are moving in a different direction. AODV routing protocol is used because it has peculiarity that routes are initiated on demand. 


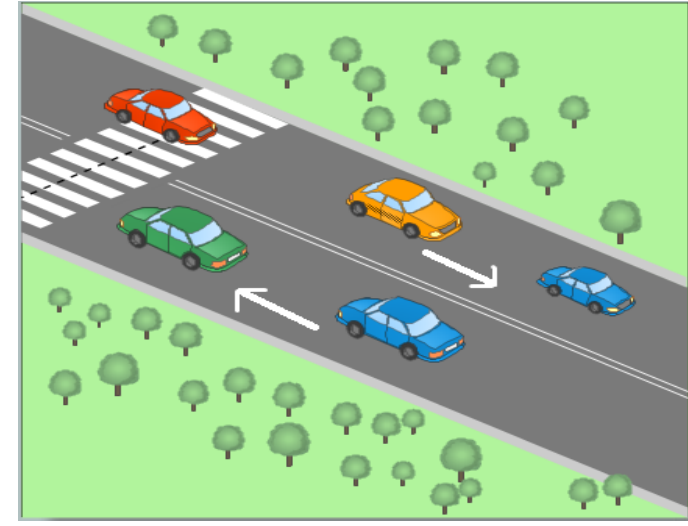

Fig 3: Highway Scenario Overview [7]

\subsubsection{City Scenario}

City scenario in the system assume that number of nodes are more than highway scenario because there are obstructions like streets, avenues and lots of intersections located near each other. Fig 4 shows the city scenario with intersections and there is need to have communication in both the direction. Information is to be spread in all direction about traffic density, mishaps etc. OLSR protocol is used in this scenario because routes are needed continuously due to high traffic density.

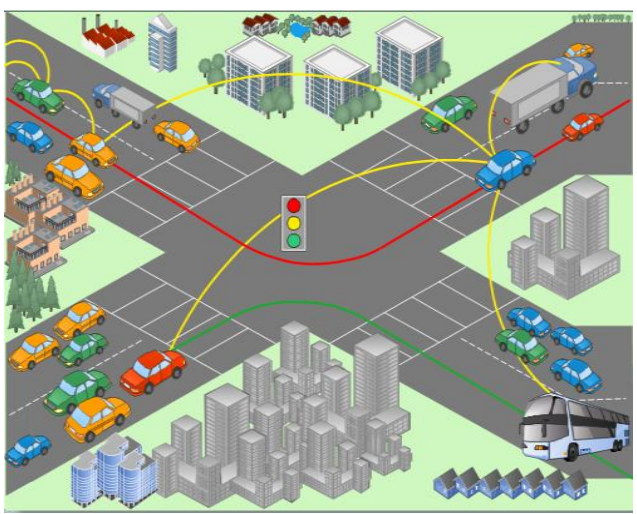

Fig 4: City Scenario Overview [7]

\subsubsection{Cluster based approach}

The cluster based approach is necessary when there is congestion in the network which creates increased overhead. This type of situation occurs when number of nodes is large for routing in network. Fig. 5 shows approach of the cluster in network. The four lanes shown in the figure have individual cluster head which is indicated in fig by different color. The cluster head is responsible for communicating outside the particular cluster.

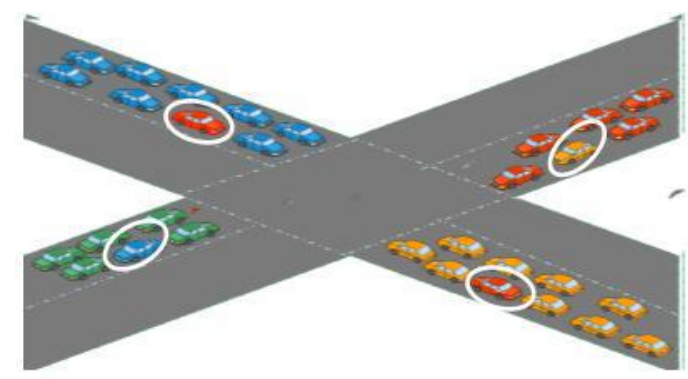

Fig 5: Cluster formation [7]

\section{DELAY TOLERANT NETWORKS (DTN)}

The Delay tolerant networks (DTN) are the networks which do not require immediate data delivery and can wait for a time period before the delivery of data. DTN use the concept of store and forward [2]. DTN network uses bundle protocol over IP network. The Bundle protocol wraps up data of applications and transfers it as a bundle to lower layers of overlay network. There may be multiple copies of a bundle simultaneously in a DTN network because of store and forward strategy [2]. Bundle can be fragmented by overlay networks if required during transmission. DTN vehicular ad hoc network uses store and forward strategy because of frequent network partitioning due to high speed of vehicles.

Due to highly dynamic nature of VANETs, it is observable to have frequent network partitioning and link failures. During the design of routing algorithm for VANETs these issues must be kept in mind. VANETs has three modes [2]: (1) Infrastructure, (2) Ad Hoc, (3) Hybrid.

In the infrastructure mode, each vehicle can communicate to each other through infrastructure unit only. In the ad hoc mode, all vehicles can communicate to each other directly without any infrastructure units. The Hybrid mode is the combination of two modes, infrastructure and ad hoc mode in which vehicles can communicate to each other directly or through infrastructure units as shown in figure 6 .
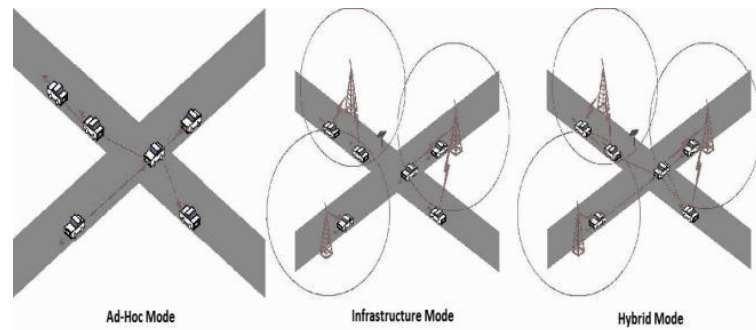

Fig 6: Different modes of VANETs [2]

\section{ROUTING ALGORITHMS FOR DTN VANET}

In the DTN, to bundle together all the information required for a transaction, minimizing the number of round-trip exchanges, which is useful when the round-trip time is very large. To help the routing and scheduling decisions, the bundles of the data follow the store-carry forward mechanisms [6].

In the delay tolerant networks (DTNs), there is no direct endto-end path between source and destination. The DTNs are defined as those networks that embrace the concept of occasionally connected networks that may suffer from frequent partitions [6]. In a real situation the vehicles are distributed over a wide area and move arbitrarily, and the network is easily partitioned. These characteristics of vehicular networks are similar to DTNs. So vehicular networks can be treated as the DTNs and defined as the vehicular delay tolerant networks (VDTNs) [6].

The routing protocols in the VANETs aim to establish an endto-end connectivity between network nodes, which is different from the case of the delay tolerant environment. Routing protocols in VDTNs use the store-carry-forward standard of DTNs to deliver data. This standard is based on the premise that the end-to-end network path may exist over time. The bundle protocol, which is the base of DTN, does not address 
routing problems without establishment of routes between nodes. The different DTN routing protocols are as following-

\subsection{MaxProp (Maximum Priority).}

MaxProp is a routing protocol which is designed for vehicular DTNs. The MaxProp protocol is based on store-carry-forward mechanism which is usually utilized in a DTN environment. The authors in [8] proposed an algorithm which enables nodes to assign the priority to the packets. On the basis of the given priorities, each node can decide either transmit or drop the packet. In the VDTNs, the transmission duration and opportunities for each node are limited, the nodes move fast in sparse areas. The buffer of node is limited in real environment. So to decide the priority of packets in a buffer of nodes is important when performing efficient routing. In the MaxProp, when two nodes communicate with each other, they exchange packets in specific order. If the node currently in contact is the destination node of some packets, these packets are transmitted first. Secondly, the routing information is exchanged which includes the estimated probability of meeting any node. The calculation of the probability is based on the number of encounters between two nodes. At the end, an acknowledgement of delivered to confirm that data is transmitted.

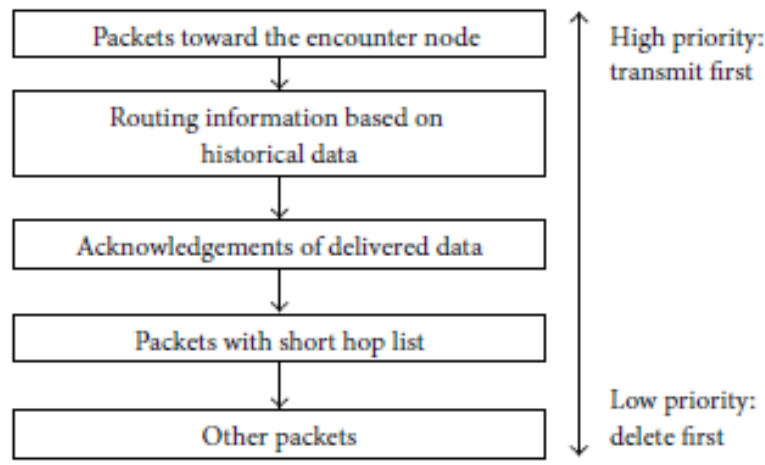

Fig 7: The priority of packet in MaxProp [8]

In MaxProp, each packet stores a hop list of nodes that the packet already traversed. This hop list enables each node to identify the age of packets. The packets with lower hop list values are considered new packets and thus higher priority is assigned to them. In case of any node found the packets with the highest priority are transmitted first and the remaining packets are transmitted later. On the other hand, the packets which have the lowest priority will be deleted first in case a buffer is full.

\subsection{PBRS (Probabilistic Bundle Relaying Scheme).}

The roadside units (RSUs) support communications between the vehicles and infrastructures. In real environments RSUs cannot cover all the roadside areas because of the deployment cost. So communications over the relaying vehicles are considered one of the solutions to support uncovered areas by RSUs. The RSU transmits its data to the incoming vehicles which enter its transmission range. PBRS [9] proposed a decision-based scheme which makes RSUs to determine whether or not to release its data to a vehicle on the basis of certain criterion. Figure 8 shows the VDTN which is considered in the PBRS. The source RSU $S$ has data to forward to the destination RSU $D$. The Vehicles passing by $S$ makes $S$ become aware of the speed of those vehicles. PBSR calculates the release probability by utilizing the speed of vehicles. When a vehicle $V i$ enters a communication range of $S$, the $S$ holds its data until the vehicle moves out of the range or a next vehicle $V i+1$ enters the coverage area. If the $V i+1$ is faster than $V i$ and $V i+1$ is considered to reach $D$ before the $V i$ does, $S$ transmits its data to $V i+1$. This technique increase the transfer rate from RSU S to RSU D.

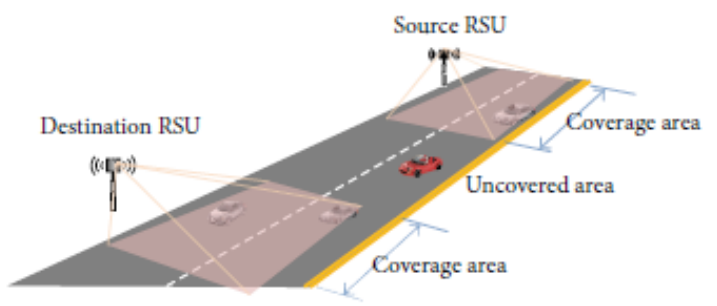

Fig 8: VDTN in PBRS [9]

\subsection{ASCF (Adaptive Carry - Store - Forward).}

ACSF assumes that RSUs cannot cover all the roadside areas like the PBRS. ACSF utilized a store-and-forward technique for relaying data. It focused on the outage time of a target vehicle in an uncovered area. A message forwarding mechanism was proposed for reducing the outage time for vehicles in the ACSF scheme [10].

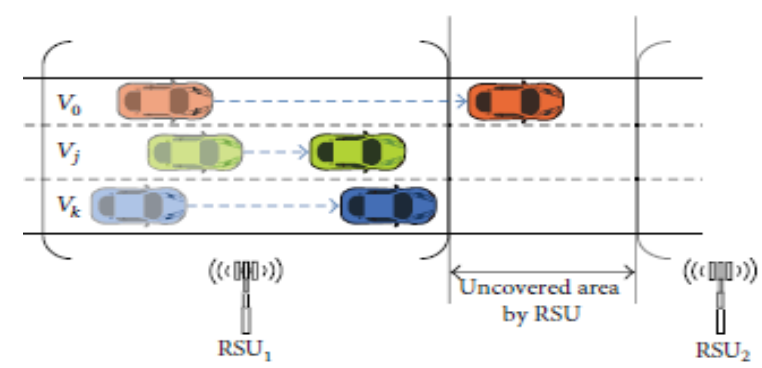

Fig 9: Communications in ASCF [10]

Figure 9 shows the deployment of vehicles and RSUs considered in ACSF. The uncovered area means the road segment which is not in the transmission range of any RSU(s). In Figure 9, it is shown that vehicles move from left to right side of the road. After the entrance of $V 0$ in the covered area of the RSU1, it starts communicating data with RSU1. $V 0$ is moving, after some time it will be entering into the uncovered area. The vehicles $V j$ and $V k$ can be used as a relay to receive the remaining data from RSU1 and forward it to $V 0$. For this reason, RSU1 selects the node which provides longer connectivity to $V 0$, thus decreasing the outage time. The outage time can be calculated by the moving speed of each node. RSU can be easily aware of its transmission range and the moving speed of nodes moving in it, RSU can calculate when $V 0$ moves out of its communication range.

Before node $V 0$ leaves the coverage area, RSU1 selects the relay node with a maximum connectivity time with $V 0$. ACSF assumes that $V 0$ is required to adjust its speed in an uncovered area for a longer connection with a relaying vehicle selected by RSU1.

\subsection{FFRDV (Fastest-Ferry Routing in DTN-Enabled VANET).}

FFRDV protocol was proposed for sparse ad hoc networks to support a highway road environment where vehicles are moving with high speeds and few traffic lights [11]. In the FFRDV, the roads are divided into the logical blocks based on 
geographic information. Each vehicle can get its current location by the GPS and it broadcast its location and speed with other vehicles in the same block by hello messages. When an emergent event occurs, FFRDV selects message ferries which have the responsibility of relaying data according to velocity based strategy.

First, the vehicle which senses an event becomes an initial ferry. It selects the fastest vehicle within block as a next ferry. Second, if the ferry enters in a new block it broadcasts a hello message to find a new ferry. Any nodes, which are able to receive a new data, send a response message, including their current speed. The ferry node compares the speeds and finds the fastest vehicle. If it is faster than itself, it sends the data to node or it holds the data. This mechanism is performed repeatedly block by block.

\subsection{DARCC (Distance-Aware Routing with Copy Control).}

The routing decision determines how to replicate or forward message copies to the suitable nodes. DARCC applies this concept of DTN routing to vehicular environments [12]. The vehicles in the DARCC determine whether to transmit data or not to their encountering vehicles with 2 principles. If location of the destination of data is available, the data is forwarded to the vehicle which is closer to the destination. Otherwise, DARCC prefers spreading the data to different direction to increase the probability to meet destination. Figure 10 shows the concept of DARCC, where each vehicle is equipped with a GPS, thus the vehicle can calculate its current motion vector. The motion vector is the speed of vehicle and its moving direction. The vehicle $A$ turns left in junction during certain time $t$, then its motion vector of time $t$ is calculated like arrow in the Figure 10. Each vehicle periodically broadcasts a beacon message including its location, current motion vector, and the list of the messages it has. If the vehicles are moving in different directions, the replication helps to perform the successful delivery, because the other vehicles may reach its destination on its way before the source. Thus, the vehicles $A$ and $B$ replicate their packets to each other, respectively.

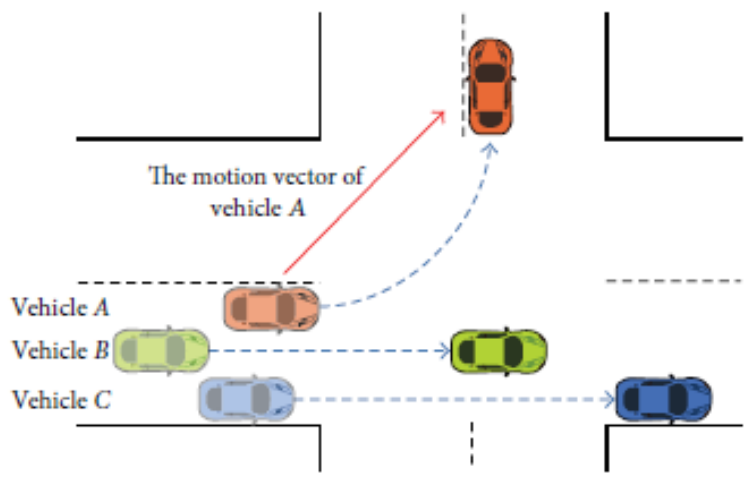

Fig 10: Concept of DARCC [12]

\subsection{DAWN (Density Adaptive Routing with Node Awareness).}

The DAWN [13] assumes an urban sensing application. As shown in Figure 11, there are $N$ fixed sensor in roadside, and one base station to collect the data. The sensors are regularly deployed and the base station is located at the center of the network area. The data packets are generated at the sensors, and each packet includes its origin location and generation time. The vehicles and mobile nodes are more like travelling in the random cells. When the vehicle moves into new cell they collect the data packet from sensors and store it in its buffer. If two vehicles meet, they replicate their packets to each other.

The data forward strategy is decided by the density of the cell. If density is low the forward strategy is the same as epidemic, that is, a node replicates all the data it has to encounter nodes. If the density of cell increases, the throughput is restricted by congestion due to the limitation of wireless channel capacity. So the UIV (utility incremental value) is proposed in DAWN to give priorities to the packets. The packets with higher UIVs should be transmitted with higher priority (Figure 11). The UIV is calculated by each node to maximize the probability of packets that are delivered to the base station before deadline.
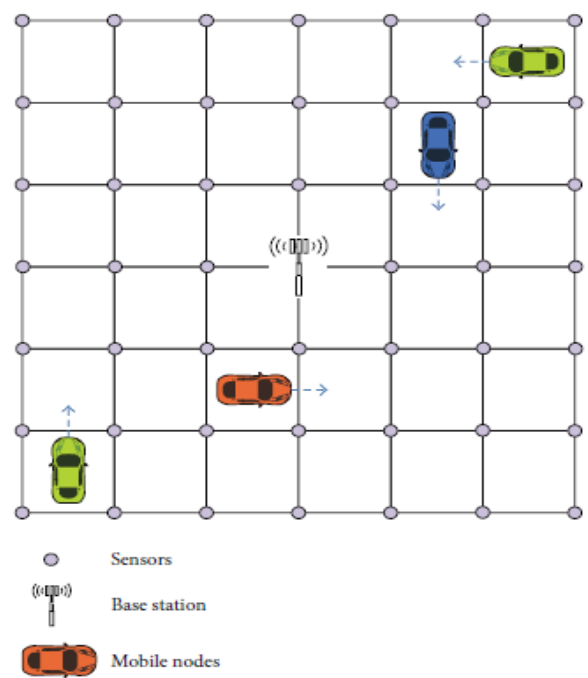

Fig 11: Network model in DAWN [13]

\subsection{GeOpps (Geographical Opportunistic Routing).}

GeOpps routing includes the location information of the vehicle into account. Geographical opportunistic routing for vehicular networks (GeOpps) aims to enhance the performance of single-copy routing protocol in VDTNs [14]. It uses the geo location of vehicles to forward geographical bundle opportunistically towards the final destination location. The vehicle that is heading towards or near the destination location of the bundle becomes the next bundle carrier. The closest point where a vehicle carries the bundle is called nearest point and used to compute minimum estimated time of delivery (METD).

A vehicle with the lowest METD is the candidate bundle forwarder/carrier. GeOpps assumes that the bundle carrier always find another vehicle when it arrives at the nearest point. GeOpps assigns weights according to varying speed of vehicles and their remaining distances to the nearest points. It does not provide a method to optimally calculate these weights.

\subsection{GeoSpray (Geographical Spray in VDTN).}

GeoSpray algorithm [15] uses the principles of single-copy single-path GeOpps to perform multi copy multipath bundle routing approach. Multi copy routing schemes are used for their high delivery ratios, low bundle delivery delays, and high overheads due to duplicated copies. GeoSpray adopts the 
replication approach of the spray-and-wait protocol [15] to limit the number of copies. Initially, it uses a multiple copy scheme, which spreads limited copies of the bundle to exploit diverse paths. After this, it switches to a single-copy forwarding scheme. GeoSpray removes the delivered bundles from vehicles' storage by propagating the delivery information. As a result, it get better delivery ratio than GeOpps at the cost of high replication overhead.

\subsection{Location and Direction Aware Opportunistic Routing (LDAOR).}

The LDAOR [16] method proposed for the opportunistic VANET to improve the performance of routing. The physical location is considered and direction of vehicles for choosing the best forwarder node among multiple neighbor nodes. LDAOR can provide better performances compared to other conventional routing protocols even when resources are limited and traffic density is high. The LDAOR reduces traffic loss, aborted messages, and overhead ratio. It increases the probability of successful message delivery. The LDAOR provides smaller end-to-end delay at low traffic loads. Although the delivery ratio and overhead in LDAOR is not significantly different from MaxProp, but the differences between LDAOR and MaxProp in terms of end-to end delay, loss in buffers and aborted messages are considerable. The complexity of LDAOR depends on the number of neighbor nodes in each contact.

\section{CONCLUSIONS}

The vehicular networks are characterized by a highly dynamic network topology, disruptive and intermittent connectivity. In such kind of network environments, a complete path from source to destination does not exist on the most of the time. Vehicular delay-tolerant network (VDTN) architecture was introduced to deal with this type of connectivity constraints. VDTN assumes asynchronous, bundle-oriented communication, and a store-carry-and-forward routing paradigm. A routing protocol for the VDTNs should make the best use of the tight resources available in network nodes to create a multi-hop path that exists over time. In this paper, we have performed a detailed survey of recent developments in VANET, VANETs architecture, communication types, vehicular DTNs, Different routing algorithms. Finally with this paper, we aim to motivate further research interest for existing routing constraints in VDTNs.

\section{REFERENCES}

[1] Ramin Karimi, Norafida Ithnin ,Shukor Abd Razak, Sara Najafzadeh, "DTN Routing Protocols for VANETs: Issues and Approaches", IJCSI International Journal of Computer Science, Issues, Vol. 8, Issue 6, No 1, November 2011.

[2] Arun Kumar, "Enhanced Routing in Delay Tolerant Enabled Vehicular Ad Hoc Networks", International Journal of Scientific and Research Publications, Volume 2, Issue 9, September 2012.

[3] Manjunath P S, Narayana Reddy, "A Review on Security and Challenges for Vehicular Ad Hoc Networks", International Journal of Emerging Technology and Advanced Engineering, Volume 4, Issue 5, May 2014.

[4] Wenshuang Liang, Zhuorong Li, Hongyang Zhang, Shenling Wang, and Rongfang Bie, "Vehicular Ad Hoc Networks: Architectures, Research Issues, Methodologies, Challenges, and Trends", Hindawi
Publishing Corporation, International Journal of Distributed Sensor Networks, Volume 2015.

[5] J. Yang and Z. Fei, "Broadcasting with prediction and selective forwarding in vehicular networks," International Journal of Distributed Sensor Networks, vol. 2013, Article ID 309041, 2013.

[6] Hyunwoo Kang, Syed Hassan Ahmed, Dongkyun Kim, and Yun-Su Chung, "Routing Protocols for Vehicular Delay Tolerant Networks: A Survey", Hindawi Publishing Corporation, International Journal of Distributed Sensor Networks, Volume 2015.

[7] Shubhrant Jibhkate, Smith Khare, Ashwin Kamble, Amutha Jeyakumar, "Advanced Adaptive Routing Algorithm for Highway and City Scenarios in VANET", International Journal of Innovative Research in Computer and Communication Engineering, Vol. 3, Issue 3, March 2015.

[8] J. Burgess, B. Gallagher, D. Jensen, and B. N. Levine, "MaxProp: routing for vehicle-based disruption-tolerant networks", Proceedings of the 25th IEEE International Conference on Computer Communications (INFOCOM '06), vol. 6, pp. 1-11, Barcelona, Spain, April 2006.

[9] M. J. Khabbaz, W. F. Fawaz, and C. M. Assi, "Probabilistic bundle relaying schemes in two-hop vehicular delay tolerant networks," IEEE Communications Letters, vol. 15, no. 3, pp. 281-283, 2011.

[10] D. Wu, G. Zhu, and D. Zhao, "Adaptive carry-store forward scheme in two-hop vehicular delay tolerant networks," IEEE Communications Letters, vol. 17, no. 4, pp. 721-24, 2013.

[11] D. Yu and Y.-B. Ko, "FFRDV: fastest-ferry routing in DTN enabled vehicular Ad Hoc networks," Proceedings of the 11th International Conference on Advanced Communication Technology (ICACT '09), pp. 14101414, February 2009.

[12] W. Z. Lo, J. S. Gao, and S.C. Lo, "Distance-aware routing with copy control in vehicle-based DTNs," in Proceedings of the IEEE 75th Vehicular Technology Conference (VTC '12), pp. 1-5, IEEE, June 2012.

[13] Q. Fu, L. Zhang, W. Feng, and Y. Zheng, "DAWN: a density adaptive routing algorithm for vehicular delay tolerant sensor networks," Proceedings of the 49thAnnualAllerton Conference on Communication, Control, and Computing, pp. 1250-1257, Monticello, Ill, USA, 2011.

[14] I. Leontiadis and C. Mascolo, "GeOpps: geographical opportunistic routing for vehicular networks," in Proceedings of the IEEE International Symposium on a World of Wireless, Mobile and Multimedia Networks (WOWMOM'07), pp. 1-6, June 2007.

[15] F. Warthman, "Delay-Tolerant Networks (DTNs) A Tutorial”, DTN Research Group Internet Draft, 2003.

[16] Marziyeh Barootkar, Akbar Ghaarpour Rahbar, and Masoud Sabaei, "LDAOR Location and Direction Aware Opportunistic Routing in Vehicular Ad hoc Networks", Journal of Telecommunication and Information Technology, 1, 2016. 